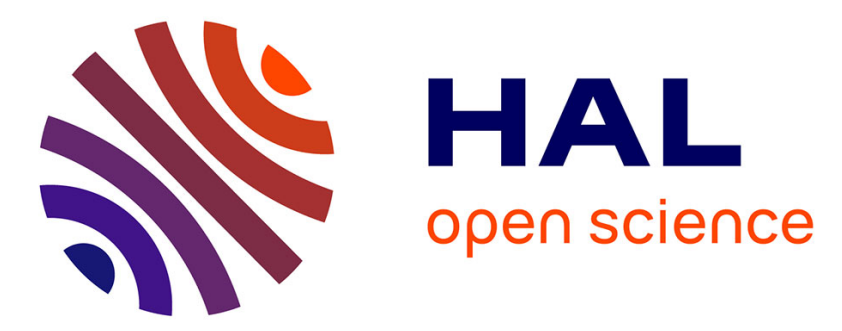

\title{
Towards sound absorption in a cylindrical lined duct using CFD with time-domain impedance boundary condition
}

Loris Casadei, Thomas Node-Langlois, Hugues Deniau, Estelle Piot, Cyril Polacsek

\section{To cite this version:}

Loris Casadei, Thomas Node-Langlois, Hugues Deniau, Estelle Piot, Cyril Polacsek. Towards sound absorption in a cylindrical lined duct using CFD with time-domain impedance boundary condition. AIAA Aviation 2021 Forum, Aug 2021, Virtual event, United States. pp.AIAA 2021-2297, 10.2514/6.2021-2297 . hal-03327703

\section{HAL Id: hal-03327703 \\ https://hal.science/hal-03327703}

Submitted on 27 Aug 2021

HAL is a multi-disciplinary open access archive for the deposit and dissemination of scientific research documents, whether they are published or not. The documents may come from teaching and research institutions in France or abroad, or from public or private research centers.
L'archive ouverte pluridisciplinaire HAL, est destinée au dépôt et à la diffusion de documents scientifiques de niveau recherche, publiés ou non, émanant des établissements d'enseignement et de recherche français ou étrangers, des laboratoires publics ou privés. 


\title{
Towards sound absorption in a cylindrical lined duct using CFD with time-domain impedance boundary condition
}

\author{
Loris Casadei* and Thomas Nodé-Langlois ${ }^{\dagger}$ \\ Airbus S.A.S, Toulouse, France, 31060 \\ Hugues Deniau ${ }^{\ddagger}$ and Estelle Piot ${ }^{\S}$ \\ DMPE, ONERA, Université de Toulouse, France, F-31055 \\ Cyril Polacsek II \\ DAAA, ONERA, Université de Paris Saclay, Châtillon, France, F-92322
}

\begin{abstract}
Noise pollution is a major concern for aircraft manufacturer during design and certification. Very high sound levels are recorded in take-off phases upstream of the engine fan, where multitonal noise components can appear (so-called Buzz Saw Noise) and contribute in generating a complex acoustics content. Nonlinear waves propagation and modal decomposition are two fundamental aspects that must be taken into account. In this context, Time-Domain Impedance Boundary Conditions (TDIBC) are then necessary when gathering noise source generation, nonlinear propagation and liner attenuation in the same numerical simulation of a nacelle inlet. A first validation of such TDIBC has already been carried out by the authors on a 2D benchmark case. In the present work, the boundary condition will be extended to a more realistic 3D cylindrical configuration, with focus on the acoustic modal content and its attenuation with and without flow.
\end{abstract}

\section{Nomenclature}

$\mathcal{A}=$ characteristic wave amplitude

$\mathcal{A}_{m n} \quad=$ pressure wave modal amplitude

$a, b=$ coefficients of EHR (Extended Helmholtz Resonator) model

$\mathcal{B}=$ scattering operator

$c, c_{0} \quad=\quad$ speed of sound (local, static)

$f \quad=$ frequency

$\mathcal{F} \quad=$ sum of characteristic variables fluxes

$h_{i} \quad=$ auxiliary function

$I \quad=$ acoustic intensity

$i \quad=$ imaginary unit $(\sqrt{-1})$

$k=$ wavenumber in modal decomposition

$k_{r, m n}=$ radial wavenumber in modal decomposition

$k_{z, m n}=$ axial wavenumber in modal decomposition

$l_{c} \quad=$ length of liner cavity

$l, L_{\tau} \quad=$ fictitious $1 \mathrm{D}$ space dimension, $1 \mathrm{D}$ distance covered in delay advection

$\mathscr{L}, \mathcal{D}, \mathcal{T}=$ convective normal, diffusive, convective transverse flux contributes of characteristic variables

$M \quad=$ Mach number

$m, n=$ azimuthal, radial order in modal decomposition

$N_{\xi}, N_{s}=$ number of poles (diffusive, oscillatory)

$p, p_{0} \quad=$ pressure (local, static)

\footnotetext{
*Ph.D. Student, Acoustics Methods, loris.1.casadei@airbus.com, AIAA member 1192275

†Aeroacoustics Numerical Simulation Expert, Acoustics Methods, thomas.node-langlois@airbus.com

$\doteqdot$ Dr. Engineer, hugues.deniau@ onera.fr

${ }^{\S}$ Dr. Engineer, estelle.piot@onera.fr

"ISenior Research Engineer, cyril.polacsek@ onera.fr
} 


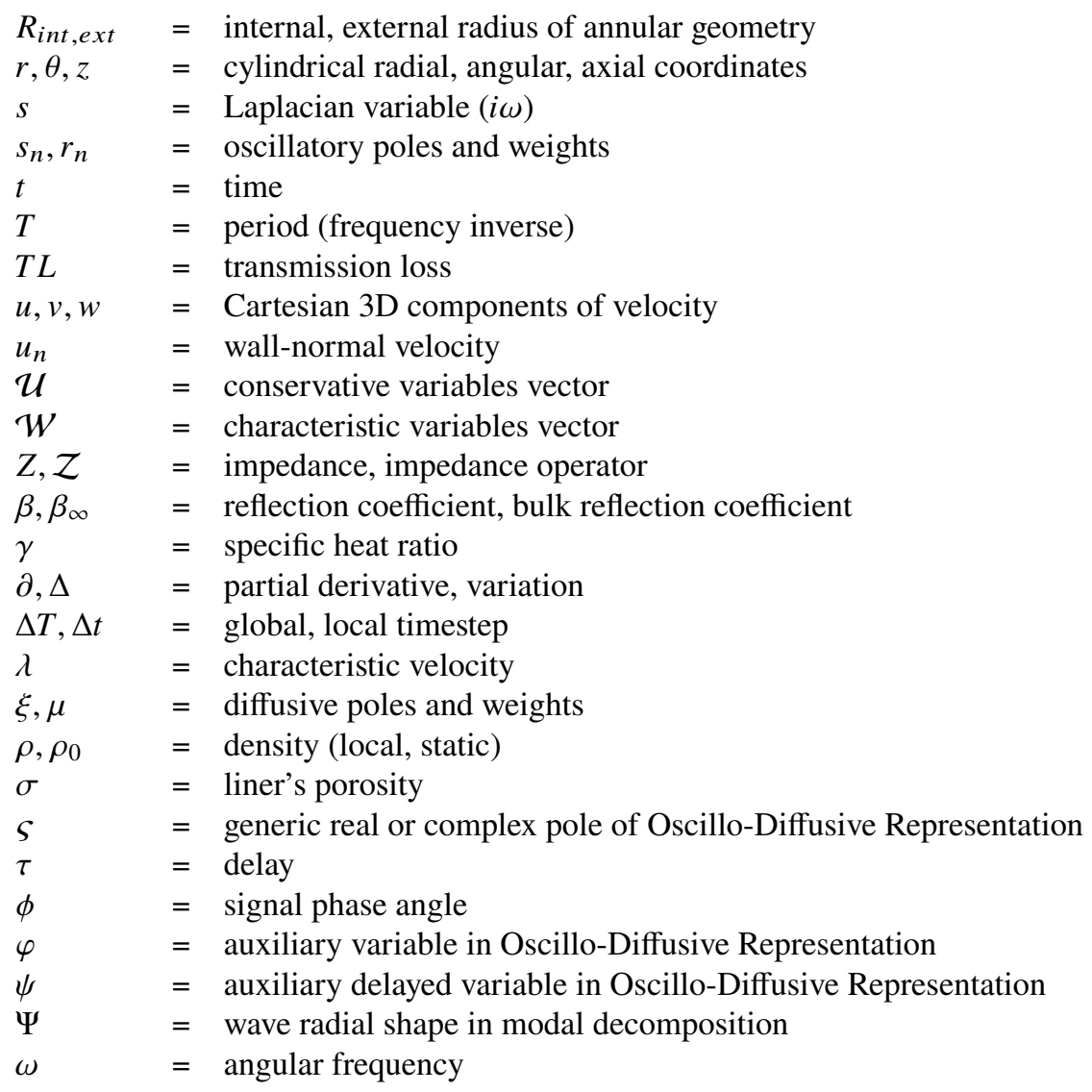

\section{Introduction}

In modern times, the environmental impact of civil aerial transports is a crucial topic worldwide. This includes obviously carbon and pollutant emissions, but also other aspects such as acoustic annoyance in the airport surroundings and cabin. With the attention focused on the noise generated by the engine, take-off and landing are two critical phases to be properly tackled due to their high sound levels. One of the most efficient solution in turbofan acoustic problems is to cover specific engine nacelle parts with sound-absorbing materials called "liners". The typically used layout in aeronautics is the Single Degree Of Freedom (SDOF), consisting of an array of honeycomb cavities backed by a rigid plate on a side and a perforated plate on the opposite one. Variations of this liner have been developed to optimize specific aspects: the Wiremesh SDOF, adding a wiremesh layer to decrease the sensitivity to the grazing flow, or the Double Degree Of Freedom (DDOF) liner, composed by two series of honeycomb cavities of different heights and separated by a porous septum, extending the spectrum range of acoustic absorption. The geometry can be tuned (in terms of cavities' length or width, plate's width and holes' diameter) to obtain a specific response. In order to maximize the noise reduction benefit of the treatment, an accurate numerical approach is fundamental to support its design.

Sound absorption is by definition a phenomena belonging to the frequency domain. Translating this effect in a time-domain formalism would make possible its implementation in classical unsteady Computational Fluid Dynamics (CFD), time-domain Computational AeroAcoustics (CAA) and coupled CFD/CAA tools. Time-domain formulations of acoustical liners are as much appealing as complex to handle. Direct Numerical Simulation (DNS) for real liner geometries mounted in a turbofan model is far from being applicable on an industrial scale, due to the high grid discretization accuracy needed to correctly resolve the unsteady field in the geometric details, as investigated in [1] with the use of Lattice Boltzmann method. Time-Domain Impedance Boundary Conditions (TDIBC) have been developed in the last two decades in order to numerically model the sound absorption mechanism of a lined wall at a lower numerical cost and to be coupled to a classical time domain CAA solvers. First attempts emerged with the work of Tam and Auriault [2], whose three-parameters model have been widely used for its implementation simplicity, however backed by a limitation in the range of applicability. Different models have followed, which can be divided into two categories: based 
on physical parameters or on numerical multi-poles schemes. The first ones are commonly rational multi-parameter, single polynomial or fractional models characterized by coefficients linked to the liner's physics (resistance, reactance, ...), as in [2, 3]. The second ones are pure numerical models, sum of elementary dynamical systems of first and second order obtained through mathematics approximations, hence losing physical meaning but easy to translate in time-domain. Some examples are in [4, 5]. Their main problem is found to be the need of solving numerically expensive convolution products and storing an accumulator. Models combining physics and multi-poles emerged in the last decade [6, 7]. In particular, the recent work of Monteghetti [8, 9] provides a reliable modelling based on a mathematical technique referred to as the Oscillatory-Diffuse Representation (ODR), which adopts auxiliary differential equations to model the lined wall effect and relies directly on the liner's geometry. This approach is currently validated for linear liners, where the impedance is independent of the variations in acoustic level and flow speed. Preliminary studies from the authors have investigated how to extend the model to the nonlinear response of the material, without detailed validation at this stage.

Another point of debate in the literature when speaking of acoustic impedance is which formulation to adopt: impedance $Z$ [2, 3, 10, 11], admittance $Y$ [12-14], or reflection coefficient $\beta$ [8, 9, 15]. Gabard et al. [10] suggest the use of a $\beta$-formalism to avoid mode instabilities. In fact, $\beta$ is a continuous function, without asymptotic-like singularities in Bode's diagrams, and bounded in amplitude in the range [0,1]. It has been demonstrated in [16] and further confirmed by Monteghetti [8] how using the $\beta$-formulation in a CAA simulation guarantees a CFL stability condition independent on the value of the reflection coefficient, which is not true for a $Z$-formulation, thus making it a reasonable choice for large scale numerical applications. TDIBCs have been initially developed in the framework of CAA, through the simulation of Euler Equations with a Linearized (LEE) or Non-Linearized (NLEE) form, as first studied at ONERA in [17-19]. However, implementing them in a CFD solver is a challenging task. The interest in having a coupled CFD numerical tool with a TDIBC is that it allows to take into account complex flow features while modeling the sound absorption of the liner. Furthermore, a single time-based numerical simulation would be sufficient, without need of separately analyzing the liner's behaviour with CAA tools, hence drastically reducing the workload simplifying the coupled CFD/CAA process. A further interest of a TDIBC in a CFD solver is the possibility to account simultaneously for sound absorption and nonlinear waves propagation in presence of a boundary layer. An industrial application which would profit of all these advantages, is the numerical simulation of the shock waves generated by a transonic rotor and propagated through an acoustically treated 3D inlet. The ODR method provides an attractive approach in this context, thanks to its adaptability to complex liner geometries and broadband frequency applications. From a design perspective of aero-engine and aircraft manufacturers, its capability to simulate the attenuation of nonlinear broadband noise phenomena makes it a preferred choice when building an industrial numerical tool.

First promising results have been achieved in [20] for impedance laws with a simple dynamics, such as the damped Helmholtz oscillator, on a multi-pole model applied to a delayed reflection [15]. A Finite-Volume (FV) approach has been tested in [21], where a CFD/CAA Linearized Navier-Stokes code and a Large Eddy Simulations (LES) code had implemented the impedance condition of [2]. However, these demand either a cumbersome formulation relying on two solvers or heavy calculation times due to the fine grid requirements of a LES. A successful implementation of the fully broadband ODR model has been recently achieved with a Spectral-Difference CFD code [22, 23]. Its high-order spatial scheme proved to be ideal in the discretization of such time-domain model. The recent work from Shur et al. [24] is closely aligned to the present development. A purely numerical multi-pole TDIBC model is derived as extension from Dragna's [11] in order to include turbulence fluctuations and sound level dependency on the impedance law, avoiding the expensive convolution process with use of auxiliary functions. Further studies on TDIBC are carried on nowadays by Naïr et al.in [25] for industrial purpose, with implementation of a purely numerical time-domain impedance model on the time-based CAA Discountinuous-Galerkin solver Actran-DGM. In collaboration with the authors, the same 3D benchmark case here analysed is tackled in [25], providing valuable additional validation resources for both parts.

In this paper, a further step regarding the TDIBC implementation in a CFD solver is addressed. It represents the continuation of the previous work from the authors [26] to implement the Oscillo-Diffusive Representation and its TDIBC model in a CFD code for simulating sound absorption of aeronautical-type liners. A Navier-Stokes Characteristics Boundary Condition (NSCBC) type of acoustic treatment has been implemented with an ODR in the elsA code, jointly owned by ONERA, Safran and Airbus, and by considering Euler and URANS equations with a structured FV algorithm. The aforementioned ODR-TDIBC is applied to an industrial benchmark study on acoustic modes attenuation in a 3D cylindrical geometry, thus including azimuthal and radial modes propagation. Compared to the previous work performed in [26], this is a more realistic numerical representation of the physical environment of engine nacelles, as also recently 
studied by Zaabar in [27], where inlet and outlet boundary conditions for modes analyses in axisymmetric ducts are discussed. Similarly to the latter and inspired by the pioneer work of Giles [28] with Euler approximation, a suitable acoustic source term injection is coupled to quasi-non-reflective NSCBC inlet and outlet boundary conditions, aiming to introduce plane waves or annular duct modes as well as strong nonlinear sound waves, either given with an analytical expression or a mapped field. Even if $\mathrm{N}$-waves are not subject of this analysis, the capability of handling very high sound levels involving nonlinear effects is validated for future applications.

The paper is structured as follows. Section $\Pi$ begins with the explanation of the mathematical tools used in the impedance definition under an ODR formalism. Then, a short description of the TDIBC implementation is given, followed by an overview on the source-input modeling and modal analysis. Section III presents the results achieved, starting with the impedance law discretization of two industrial liners. These are then used in 3D CFD simulations for predicting the attenuation of high order acoustic modes at selected frequencies and different flow configurations. The conclusive Section IV] summarizes the main achievements, foreseeing the upcoming work on the topic.

\section{Mathematical formulation}

This Section focuses on giving the theoretical background and mathematical tools for understanding the present developments. A reflection coefficient approach is used rather than impedance-based, as previously stated to be more convenient when dealing with time-domain transformations. An introduction to NSCBC theory is given in Section III.A based on the original work of [29], followed in Section III.B by fundamental equations of the Oscillo-Diffusive Representation (ODR) and its associated TDIBC model as derived in [8, 9]. Its numerical implementation in elsA code is shortly resumed in Section [I.C. and additional information can be found in the previous work from the authors [26]. Details on the other numerical boundary conditions used during this study (such as inlet and outlet) are given in Section II.D This includes generalities on acoustic modal analysis for generic high-order modes injection.

\section{A. Navier-Stokes Characteristics Boundary Conditions}

Firstly introduced by Poinsot and Lele [29], NSCBC are a valid alternative for boundary conditions of hyperbolic systems. Specifically, for acoustics problems, Rienstra [30] suggested the advantage of using characteristics forms, allowing an analytical solution to problems at high sound amplitudes (where nonlinearities appear) and determining optimal discretization schemes and stability conditions. The focus of NSCBC is on the waves crossing the boundary, solving local 1D Linearized Euler Equations (LEE), also called LODI (Linear One-Dimensional Inviscid) problem. Although this is a crude simplification making the problem non-physical, it can be used practically to manage the outgoing and incoming waves amplitude through a dedicated coupling with the Navier-Stokes equations system. When using a characteristics formalism, five waves can be defined crossing each boundary. If we define with $\mathcal{U}$ the conservative variables vector:

$$
\mathcal{U}=\left[\rho, \rho u, \rho v, \rho w, \rho\left(e+|u|^{2} / 2\right)\right]^{\top}
$$

and we consider a boundary normal to $x_{1}$ direction (in relation to Figure 11, characteristic waves amplitude $\mathcal{A}$ is linked to characteristic velocities $\lambda$ (i.e. the eigenvalue diagonal matrix) by the transportation matrix $\mathbf{P}_{\mathcal{U}}$ from conservative to characteristic variables following the relation:

$$
\mathcal{A}=\lambda \mathbf{P}_{\mathcal{U}}^{-1} \frac{\partial \mathcal{U}}{\partial x_{1}}
$$

For compactness reasons, such system is not here detailed. Additional information on its resolution on a generalized coordinate system can be found in [22, 23, 26, 29]. The sign of $\lambda$ determines the direction of the associated characteristic wave: if positive the wave leaves the domain, if negative it enters the domain and further information on it is required. These five velocities can be written as:

$$
\left\{\begin{array}{l}
\lambda_{1}=\lambda_{2}=\lambda_{3}=u \\
\lambda_{4}=u+c \\
\lambda_{5}=u-c
\end{array}\right.
$$

where $\lambda_{1}$ stands for the convective entropy wave, $\lambda_{2,3}$ for the convective rotational (tangential) waves, and $\lambda_{4,5}$ for the outgoing and incoming acoustic waves (with respect to the notations used in the right side of Figure 11. The amplitude of acoustic waves, under the assumption of LEE as adopted in the LODI problem, can be defined as:

$$
\mathcal{A}_{4,5}=p^{\prime} \pm u^{\prime} \rho_{0} c_{0}
$$




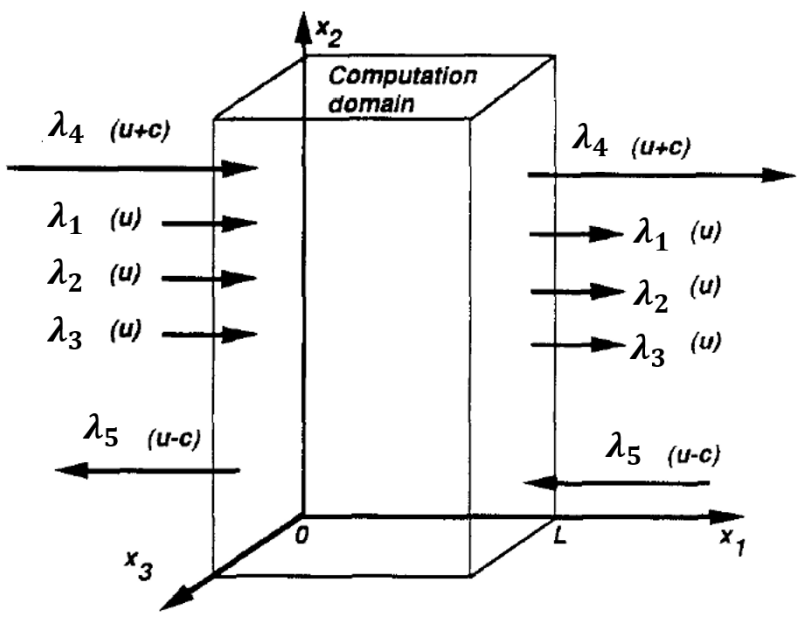

Fig. 1 NSCBC scheme - adapted from [29]

where $p^{\prime}$ and $u^{\prime}$ are the acoustic pressure and velocity (normal to the boundary) as later defined in (7). Without loss of generalities in the following, the acoustic waves will be denoted with the exponent (in), (out) for incoming and outgoing waves with respect to the fluid domain.

Full 3D Navier-Stokes equations are re-written under a characteristics formalism as sum of fluxes:

$$
\frac{\partial \mathcal{W}}{\partial t}+\mathscr{L}+\mathcal{D}+\mathcal{T}=0
$$

with $\mathcal{W}$ the characteristic variables array (obtained with the transport matrix $\mathbf{P}_{\mathcal{U}}$ from the conservative variables), $\mathscr{L}$ the characteristic waves flux related to convective term normal to the surface, $\mathcal{D}$ its related diffusive part and $\mathcal{T}$ the characteristic waves flux for tangent terms and its diffusive part. From conservation laws, the sum in (5) must be conserved across the boundary for incoming and outgoing fluxes:

$$
{\frac{\partial \mathcal{W}^{\text {out }}}{\partial t}}+\mathscr{L}^{\text {out }}+\mathcal{D}^{\text {out }}+\mathcal{T}^{\text {out }}={\frac{\partial \mathcal{W}^{\text {in }}}{\partial t}}+\mathscr{L}^{\text {in }}+\mathcal{D}^{\text {in }}+\mathcal{T}^{\text {in }}=0
$$

The latter will be used in the next sections to express the relation between incoming and outgoing acoustic waves.

\section{B. Oscillo-Diffusive Representation}

The acoustic concept called impedance characterizes the resistance and reactance of a surface material, hence its capability of reducing sound level. It is defined in the frequency domain as the ratio between pressure fluctuations $p^{\prime}$ and wall-normal velocity fluctuations $u_{n}^{\prime}$ (with the normal vector pointing inside outward the fluid domain):

$$
Z(\omega)=\frac{p^{\prime}(\omega)}{u_{n}^{\prime}(\omega)}
$$

A similar parameter, here named "reflection coefficient" $(\beta)$, can be derived from (7) and it reads:

$$
\beta(\omega)=\frac{Z(\omega)-\rho_{0} c_{0}}{Z(\omega)+\rho_{0} c_{0}}
$$

where the product $\rho_{0} c_{0}$ is the medium characteristic impedance. Regardless its natural belonging to the frequency domain, it has been demonstrated in Section 1 the convenience of translating the impedance (or reflection coefficient) in the time domain. This conversion reveals to be cumbersome, as instantaneous pressure depends on the convolution product:

$$
p^{\prime}(t)=\left[Z \star u_{n}{ }^{\prime}\right](t)=\mathcal{Z}\left[u_{n}{ }^{\prime}\right](t)
$$

where $\mathcal{Z}$ is the impedance operator. Similarly, a scattering (reflection) operator $(\mathcal{B})$ is function of the convolution product of characteristic acoustic waves amplitudes $\mathcal{A}$ :

$$
\mathcal{A}^{\text {in }}=\left[\beta \star \mathcal{A}^{\text {out }}\right](t)=\mathcal{B}\left[\mathcal{A}^{\text {out }}\right](t)
$$


Various impedance models and their time-domain translation have been proposed either to simplify or to surround the convolution problem. The Extended Helmholtz Resonator (EHR) impedance model proposed by Rienstra in [3] is a suitable impedance model when dealing with SDOF liners:

$$
Z_{E H R}(s)=\underbrace{\frac{1}{\sigma_{p}}\left(a_{0}+a_{\frac{1}{2}} \sqrt{s}+a_{1} s\right)}_{\text {perforation }}+\underbrace{\frac{1}{\sigma_{c}} \operatorname{coth}\left(b_{0}+b_{\frac{1}{2}} \sqrt{s}+b_{1} s\right)}_{\text {cavity }}
$$

where $s$ is the Laplace variable defined as $i \omega$. The parameters $a_{i}$ and $b_{i}$ are function of the liner's geometry (perforations and cavities) and can find different formulations. Herein, the coefficient sets proposed by Bruneau [31] and Crandall [32] are used as initial guess for the model, then optimized with a least-square fitting to match the experimental data set and so the "realistic" impedance law. It has been demonstrated that the impedance model (11) accepts an Oscillo-Diffusive Representation [8, 9]. With help of 88, the model in (11) is converted into a reflection coefficient formulation, then rewritten as:

$$
\beta(s)=\beta_{\infty}+h_{1}(s)+e^{-s \tau} h_{2}(s)
$$

with $\beta_{\infty}$ the bulk reflection coefficient (i.e. the frequency-independent reflection) and $\tau$ the resonance delay equal to the back-and-forth traveling time inside the liner's cavity. The functions $h_{1}$ and $h_{2}$ represent respectively the non-delayed and delayed terms, each of them defined as:

$$
h_{i}=\sum_{n \in \mathbb{Z}^{+}} \frac{r_{n}}{s-s_{n}}+\int_{0}^{\infty} \frac{\mu(\xi)}{s+\xi} d \xi
$$

The same nomenclature as in the original paper [9] has been kept, identifying with $s_{n}$ and $r_{n}$ respectively the complex conjugated pairs of oscillatory poles and weights and with $\xi$ and $\mu$ the real diffusive poles and weights. The link with the EHR model of (11) lies within the calculation of poles and weights, obtained from knowledge of the coefficients $a_{i}$ and $b_{i}$, which provide exact solution of the same model. The complete formulation after discretization reads:

$$
\beta(s)=\beta_{\infty}+\sum_{n=1}^{N_{s}} \frac{r_{1, n}}{s-s_{n}}+\sum_{k=1}^{N_{\xi}} \frac{\mu_{1, k}}{s+\xi_{k}}+e^{-s \tau}\left(\sum_{n=1}^{N_{s}} \frac{r_{2, n}}{s-s_{n}}+\sum_{k=1}^{N_{\xi}} \frac{\mu_{2, k}}{s+\xi_{k}}\right)
$$

where $N_{s}$ and $N_{\xi}$ are the number of complex conjugated oscillatory and real diffusive poles, respectively, and $\left(r_{1, n}, \mu_{1, k}\right)$, $\left(r_{2, n}, \mu_{2, k}\right)$ the weights for the non-delayed and delayed terms, respectively. Consequently, (14) needs to be converted from Laplacian (frequency) to time-domain. Instead of solving the convolution product in 9), this is done in the ODR method through additional auxiliary functions $\varphi$. The scattering operator is then given as:

$\mathcal{B}\left[\mathcal{A}^{\text {out }}\right](t)=\beta \star \mathcal{A}^{\text {out }}(t)=\beta_{\infty} \mathcal{A}^{\text {out }}(t)+\sum_{k=1}^{N_{\xi}}\left[\mu_{1, k} \varphi\left(t, \xi_{k}\right)+\mu_{2, k} \varphi\left(t-\tau, \xi_{k}\right)\right]+\sum_{n=1}^{N_{s}}\left[r_{1, n} \varphi\left(t,-s_{n}\right)+r_{2, n} \varphi\left(t-\tau,-s_{n}\right)\right]$

where the auxiliary functions $\varphi$ are solution of the ordinary differential equations:

$$
\left\{\begin{array}{l}
\partial_{t} \varphi(t, \varsigma)=-\varsigma \varphi(t, \varsigma)+\mathcal{A}^{o u t} \\
\varphi(0, \varsigma)=0
\end{array}\right.
$$

with $t>0$ and $\varsigma$ (read sigma) being either an oscillatory $\left(-s_{n}\right)$ or diffusive $\left(\xi_{k}\right)$ pole. As a result, as many auxiliary $\varphi$ functions as the number of poles of the liner discretization are added. At last, the delay effect is converted into a one-dimensional advection problem of the quantity $\varphi$ convected at the propagation speed (of sound) $c_{0}$ on a length $L_{\tau}=c_{0} \tau$, equal to the back-and-forth distance traveled in the liner's cavity (i.e. $L_{\tau}=2 l_{c}$ ), as illustrated in Fig. 2 For this purpose, a second auxiliary function $\psi$ is introduced, solution of the partial differential equation:

$$
\left\{\begin{array}{l}
\partial_{t} \psi(t, \varsigma, l)=c_{0} \partial_{l} \psi(t, \varsigma, l) \\
\psi(t, \varsigma, 0)=\varphi(t, \varsigma) \\
\psi(0, \varsigma, l)=0
\end{array}\right.
$$




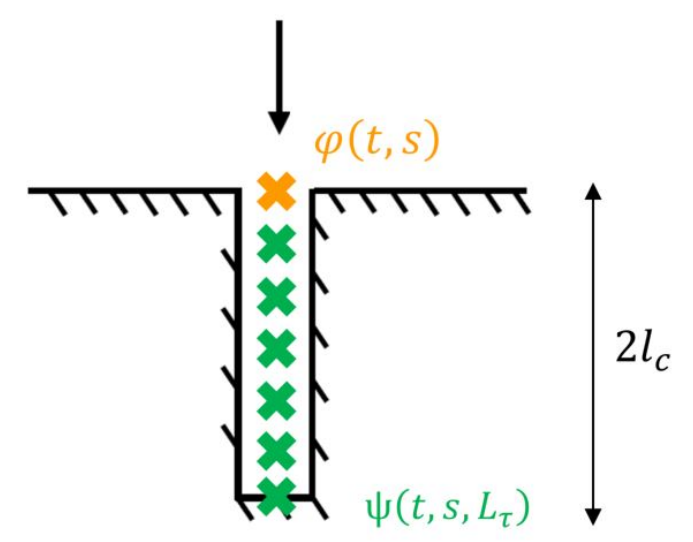

Fig. 2 Delay $1 D$ advection discretization

with $l \in\left(0, L_{\tau}\right)$ the one-dimensional spatial coordinate. Therefore, $\psi$ at the last value of the spatial array $l$ (at coordinate $l=L_{\tau}$ ) corresponds to the delayed $\varphi$ variable. The final time-domain reflection coefficient model reads:

$$
\mathcal{B}\left[\mathcal{A}^{\text {out }}\right](t)=\beta_{\infty} \mathcal{A}^{\text {out }}(t)+\sum_{k=1}^{N_{\xi}}\left[\mu_{1, k} \varphi_{\xi_{k}}+\mu_{2, k} \psi_{\xi_{k}}\right]+\sum_{n=1}^{N_{s}}\left[r_{1, n} \varphi_{s_{n}}+r_{2, n} \psi_{s_{n}}\right]
$$

where the different dependencies of $\phi, \psi$ have been dropped for brevity. The next section will give more details on how such equation is implemented in the CFD solver.

\section{TDIBC numerical implementation}

Contrarily to a classic NSCBC solid wall, where the three velocity-related characteristic waves (denoted $\lambda_{1,2,3}$ in Fig. 1) are null, a TDIBC presents a non-zero normal velocity due to acoustic interactions with the liner, reason why such velocity component must not be imposed to zero but extrapolated with LODI equations system. For standard TDIBC, incoming acoustic waves entering the domain are functions of the outgoing acoustic waves, known from the inner domain solution. Using (4), we can write the time variation of the incident (outgoing) and reflected (incoming) characteristic waves as:

$$
\left\{\begin{array}{l}
\partial_{t} \mathcal{W}^{\text {out }}=\partial_{t} p+\rho c \partial_{t} u_{n} \\
\partial_{t} \mathcal{W}^{\text {in }}=\partial_{t} p-\rho c \partial_{t} u_{n}
\end{array}\right.
$$

and then link the two waves with the linear scattering operator $\mathcal{B}$ :

$$
\partial_{t} \mathcal{W}^{\text {in }}=\mathcal{B}\left(\partial_{t} \mathcal{W}^{\text {out }}\right)
$$

By using (6), we can recast the latter into:

$$
\mathscr{L}^{\text {in }}+\mathcal{D}^{\text {in }}+\mathcal{T}^{\text {in }}=\mathcal{B}\left(\mathscr{L}^{\text {out }}+\mathcal{D}^{\text {out }}+\mathcal{T}^{\text {out }}\right)
$$

Collecting all known outgoing fluxes into $\mathcal{F}^{\text {out }}$ and expanding the scattering operator as in $(18)$, the incoming convective wave $\mathscr{L}^{\text {in }}$ in a TDIBC is computed as:

$$
\mathscr{L}^{\text {in }}=\beta_{\infty} \mathcal{F}^{\text {out }}-(\mathcal{T}+\mathcal{D})^{i n}+\sum_{k=1}^{N_{\xi}}\left[\mu_{1, k} \varphi_{\xi, k}+\mu_{2, k} \psi_{\xi, k}\right]+2 \mathcal{R e} \sum_{n=1}^{N_{s} / 2}\left[r_{1, n} \varphi_{s, n}+r_{2, n} \psi_{s, n}\right]
$$

It is reminded that diffusive terms $\left(\xi_{k}, \mu_{k}, \varphi_{\xi, k}, \psi_{\xi, k}\right)$ are real values, while oscillatory terms $\left(s_{n}, r_{n}, \varphi_{s, n}, \psi_{s, n}\right)$ are complex values. The final discretization of (22), upon a simplification of complex calculation ${ }^{*}$ gives a real value.

To be coherent with the global dual-time stepping temporal marching scheme of second-order adopted in the code, a

\footnotetext{
* Sum of complex and complex conjugate products: $a \cdot b+a^{*} \cdot b^{*}=2 \mathcal{R e}(a \cdot b)$
} 
second-order in time derivative of (16) has been discretized. If we define with $\varphi_{0}$ the variable at the current timestep and with $\varphi_{1,2}$ those previously converged in the last two iterations (i.e. at the end of the sub-iterations), the updated $\varphi_{0}$ function from a sub-iteration $j^{\prime}$ and the successive one $j^{\prime}+1$ is given by:

$$
\varphi_{0}^{j^{\prime}+1}=\varphi_{0}^{j^{\prime}}+\Delta \varphi
$$

where the time variation $\Delta \varphi$ is:

$$
\Delta \varphi\left(1-\varsigma \Delta t+\frac{3}{2} \frac{\Delta t}{\Delta T}\right)=\frac{\Delta t}{2 \Delta T}\left(-3 \varphi_{0}^{j^{\prime}}+4 \varphi_{1}-\varphi_{2}\right)+\varsigma \varphi_{0}^{j^{\prime}}-\mathcal{F}_{0}^{\text {out }} \Delta t
$$

with $\Delta T$ the global timestep and $\Delta t$ the local timestep (inherent to dual-timestep iterations). Concerning the delay treatment and the calculation of $\psi$ from (17), it corresponds to solving a 1D advection problem to "delay" the quantity $\varphi$ and obtain $\psi$ as the last value of such advection. A Spectral Difference scheme of fifth polynomial order is used to discretize the fictitious 1D cavity. The number of elements in the 1D array is chosen dependent on the maximum frequency to resolve (at least 2 elements for satisfying Nyquist criterion on the cut-off frequency) or the resolution wanted in terms of points per wavelength at a specific frequency. The chosen frequency to size up the 1D domain must imperatively be correctly resolved by the impedance model chosen for discretization (the EHR model in this case) to ensure numerical stability. In other words, it is needed that in the frequency range chosen, the reflection coefficient of the impedance model is bounded within [0,1], thus a passive function.

All the presented numerical solutions are written in a fully implicit formulation, hence the calculation of $\varphi, \psi$ and $\mathcal{F}^{\text {out }}$ is done simultaneously at the same timestep, improving robustness, numerical stability and convergence efficiency.

\section{NSCBC Inlet and Outlet with acoustic source}

In the code, an acoustic perturbation (or acoustic source) can be introduced to any NSCBC under the form of an additional pressure or pressure derivative fluctuation. A classical NSCBC inlet as derived in the original paper from Poinsot et al.[29] is used. Following the LODI theory, 4 equations are added, respectively for the three velocity components and temperature, as the missing information for enclosure of the boundary problem for a subsonic inflow condition. With supposition of an isentropic acoustic wave, the pressure fluctuation is converted into velocity or temperature fluctuation to be included in each equation. The consideration of incoming waves in a reflective inlet condition is required for taking into account how upstream waves (as for example those given by the boundary discontinuity between a hard wall and an acoustic liner) are influencing this numerical region. Concerning the outlet, only the pressure equation is specified, as this is the sole equation needed to enclose the boundary problem of a subsonic outflow condition. A similar formulation to the one of [29] is used. In this NSCBC context, pressure derivative fluctuations are added to NSCBC equations for introducing the acoustic term in the characteristic fluxes (noted $\partial \mathcal{W} / \partial t$ ). The initial approach, adopted to achieve the results shown in [26], investigated an acoustic plane wave specified at the entrance boundary, which is expressed by the following set of equations:

$$
\begin{aligned}
p & =\mathcal{A} \sin (\omega t) \\
\frac{\partial p}{\partial t} & =\mathcal{A} \omega \cos (\omega t)
\end{aligned}
$$

In order to reduce parasitic reflections appearing near the inlet and outlet boundaries, a Giles treatment [28] is implemented in the code, properly adapted to be used in a NSCBC formalism. The primitive variables (temperature and velocity for inlet or pressure for outlet) are imposed under a relaxation formalism to a time-filtered value, inspired by the work of Hixon [33]. These two solutions showed a sensible improvement in terms of stability and convergence speed. More complex sound fields, with dependency on the 3D space directions and not only axial direction as in plane waves, can be considered. A mathematical description of in-duct sound fields can be found in the book of Rienstra and Hirschberg [30]. In an annular or circular duct with hard walls, the acoustic pressure can be expressed as a sum of classical Fourier-Bessel modes. Once the thermodynamic environment is known and the instantaneous pressure measured with microphones or numerical outputs, matrix inversion techniques can provide modes amplitudes, and so their intensities and attenuation can be estimated. The circular duct case considered in this study is a simpler case of the more general annular geometry (with an internal radius null), so the set of equations is given fully for an annular 
duct. The isentropic pressure fluctuation for a modal amplitude $\mathcal{A}_{m n}$ and phase $\phi$ is given function of the cylindrical coordinates $(r, \theta, z)$ and the modal structure $\left(m, n, k_{r, m n}\right)$ as:

$$
p_{m n}^{ \pm}(r, \theta, z, \phi, t)=\mathcal{A}_{m n}\left[B_{m n} J_{m n}\left(k_{r, m n} r\right)+C_{m n} Y_{m n}\left(k_{r, m n} r\right)\right] e^{i\left(\omega t+m \theta-k_{z, m n}^{ \pm} z+\phi\right)}
$$

with:

- $(m, n)$ the azimuthal and radial orders;

- $J_{m}$ and $Y_{m}$ the Bessel functions of the first and second kind, $J_{m}^{\prime}$ and $Y_{m}^{\prime}$ their derivatives;

- $B_{m n}$ and $C_{m n}$ constants, function of the mode shape;

- $k_{r, m n}$ the radial wavenumber (real positive);

- $k_{z, m n}$ the axial wavenumber, which is real for propagative modes and imaginary for cut-off (evanescent) modes;

- \pm defining if the propagation is on the positive or negative axis.

More detailed expressions are given in Appendix. The time derivative of (27) is simply the real part of:

$$
\frac{\partial p}{\partial t}=\mathcal{R e}\left(i \omega p_{m n}^{ \pm}\right)
$$

As for the plane wave previously detailed, such time derivative is added to NSCBC equations in the characteristic fluxes $(\partial \mathcal{W} / \partial t)$. The equations above are used to define the cut-on modes at selected frequencies with prescribed amplitude injected in the numerical simulations, as considered for the test bench validations discussed in the next Section.

\section{Results and Validation}

This part is structured as follow. Section III.A presents the experimental bench used to obtain acoustic attenuation results in [34] and considered herein to validate the numerical application. The geometrical features of the industrial acoustic liners used in the experimental and present numerical studies are undisclosed, but the curves of their impedance laws are given in Section III.B, altogether with their ODR discretizations. Section III.C resumes the numerical choices for the simulations and Section III.D details the CFD results on the 3D cylindrical domain including a TDIBC, studying different frequencies, azimuthal modes and Mach numbers.

\section{A. CANNELLE test bench description}

The CANNELLE test bench has been developed by Airbus to experimentally study the sound propagation and attenuation of acoustic modes in a lined cylindrical duct, in view of aircraft nacelle design. Measurements from this bench have been recently used to present an impedance eduction technique [34]. This methodology aimed at being used in multi-modal excitation of the liner, in order to take into account multiple tones and propagating modes. However, results on acoustic modes attenuation achieved with this bench have never been published. The experimental layout is shown in Figure 3. The cylindrical bench is made of different modular parts, of which the central "Test barrel" can be acoustically treated. An acoustic source controlled by 50 loudspeakers is mounted upstream or downstream the test section, in order to simulate a bypass duct or engine nacelle inlet environment, where the acoustic perturbation is propagating along or against the flow, respectively. Figure 4 represents these two wave-traveling cases, where the black arrows indicate the flow direction, the blue waves the acoustic emission and the red surface the liner treatment. Single azimuthal modes (noted $m$ ) are excited using suited phase control on the loudspeakers and radial mode orders $(n)$ are driven by cut-off frequency, hence with no direct control on their generation. During the test campaign, up to 29 modes $(m, n)$ of azimuthal orders comprised within 0 and 9 were generated for 5 frequencies ranging between $1200 \mathrm{~Hz}$ and $3500 \mathrm{~Hz}$ and 3 Mach numbers $(0,0.3,0.6)$. Microphones were located up- and downstream the treated section to perform modal detection. This modal detection technique allowed to estimate the respective mode amplitudes and then their attenuation through the lined section. Different liners were tested in the experimental campaign. Two of them are herein considered for validating the CFD: a bypass SDOF liner Aircelle (ACL) provided by the acoustics department of Safran Nacelles, and a nacelle inlet SDOF liner designed by Airbus (AIB), with addition of a wiremesh layer glued to the perforated sheet.

\section{B. Impedance ODR of prescribed acoustical liners}

The first step when using an ODR method is to define a reliable fitting of the given impedance law over a determined frequency range. Poles and weights are computed with a MATLAB code developed in [8] for both liners at Mach 0 and 0.3. In Figures 5 and 6, comparison between the multi-pole representation (red and blue lines), the analytical EHR 

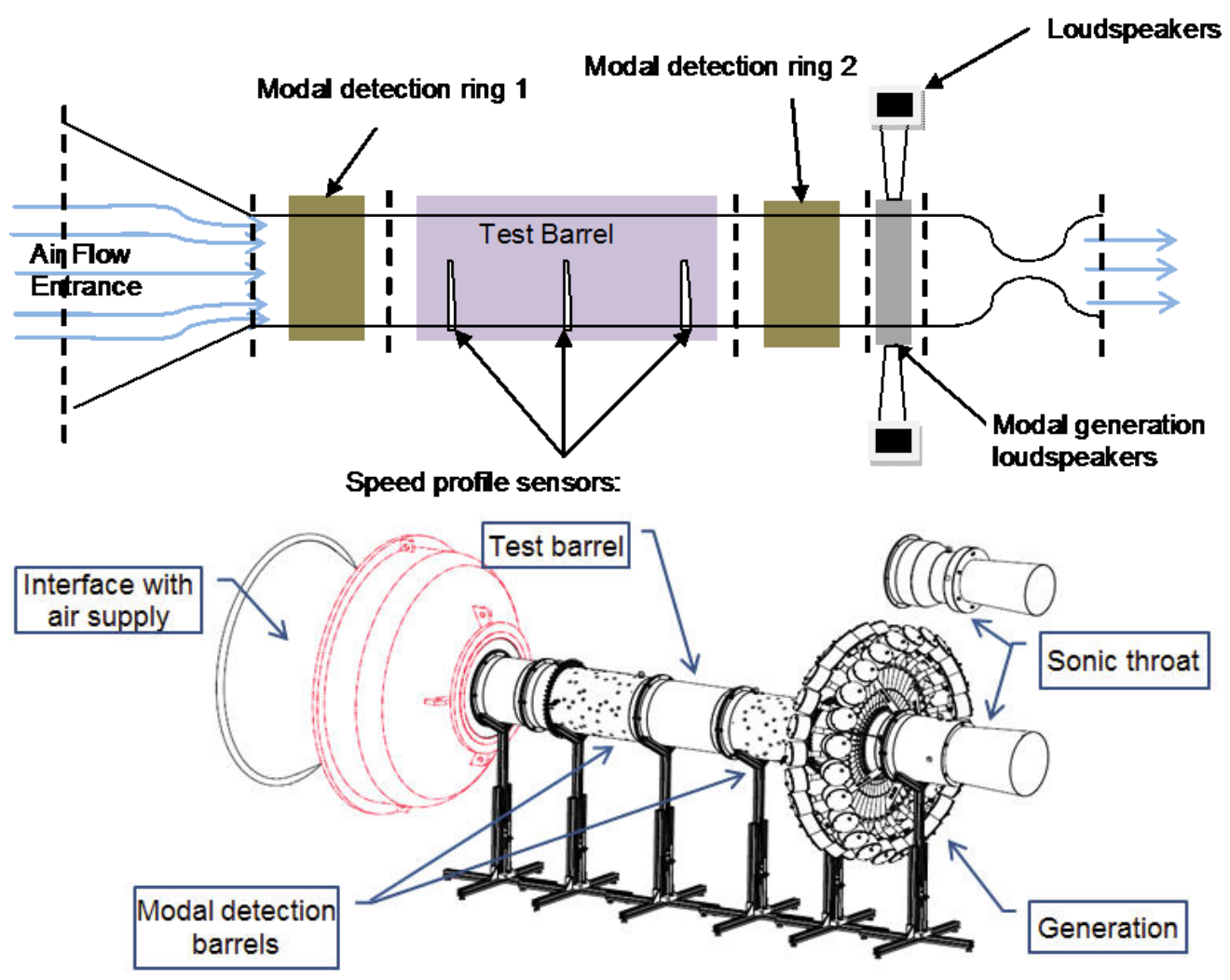

Fig. 3 CANNELLE layout - adapted from [34]

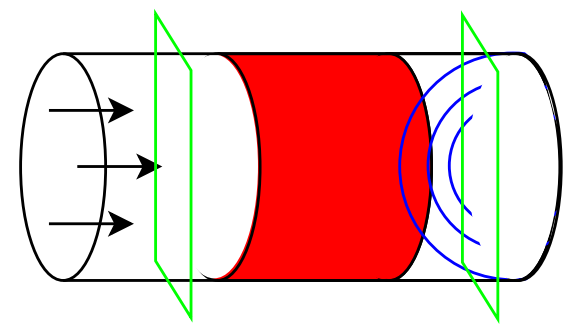

(a) Nacelle inlet

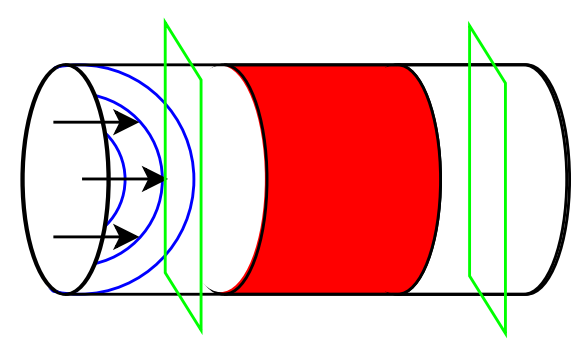

(b) Exhaust bypass duct

Fig. 4 CANNELLE test configurations

model (black dashed line) and the experimental measurements (white symbols) is shown, for respectively the AIB and ACL liners. In both figures, the bottom left and right subfigures represent respectively the reflection coefficient module and phase, i.e. the continuous function that is introduced in the CFD code as representative of the liner's physics. Three vertical dashed lines correspond to the three frequencies that are investigated in the CFD simulations (1600, 2600 and $3500 \mathrm{~Hz}$ ), in order to verify that the ODR discretization is accurate at these specific values. 

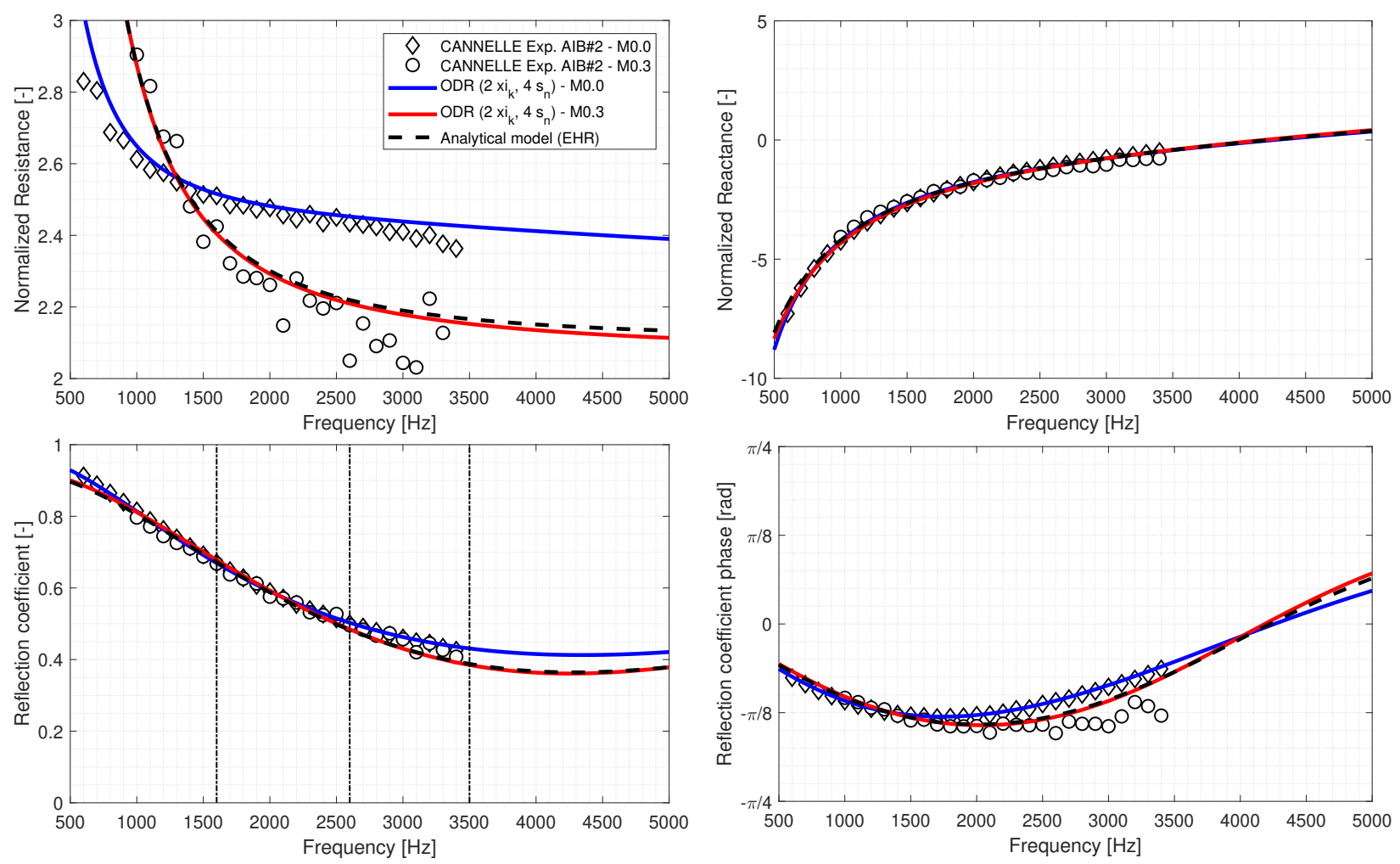

Fig. 5 Oscillo-Diffusive Representation of AIB Liner, 120dB, Mach 0.0 and Mach 0.3
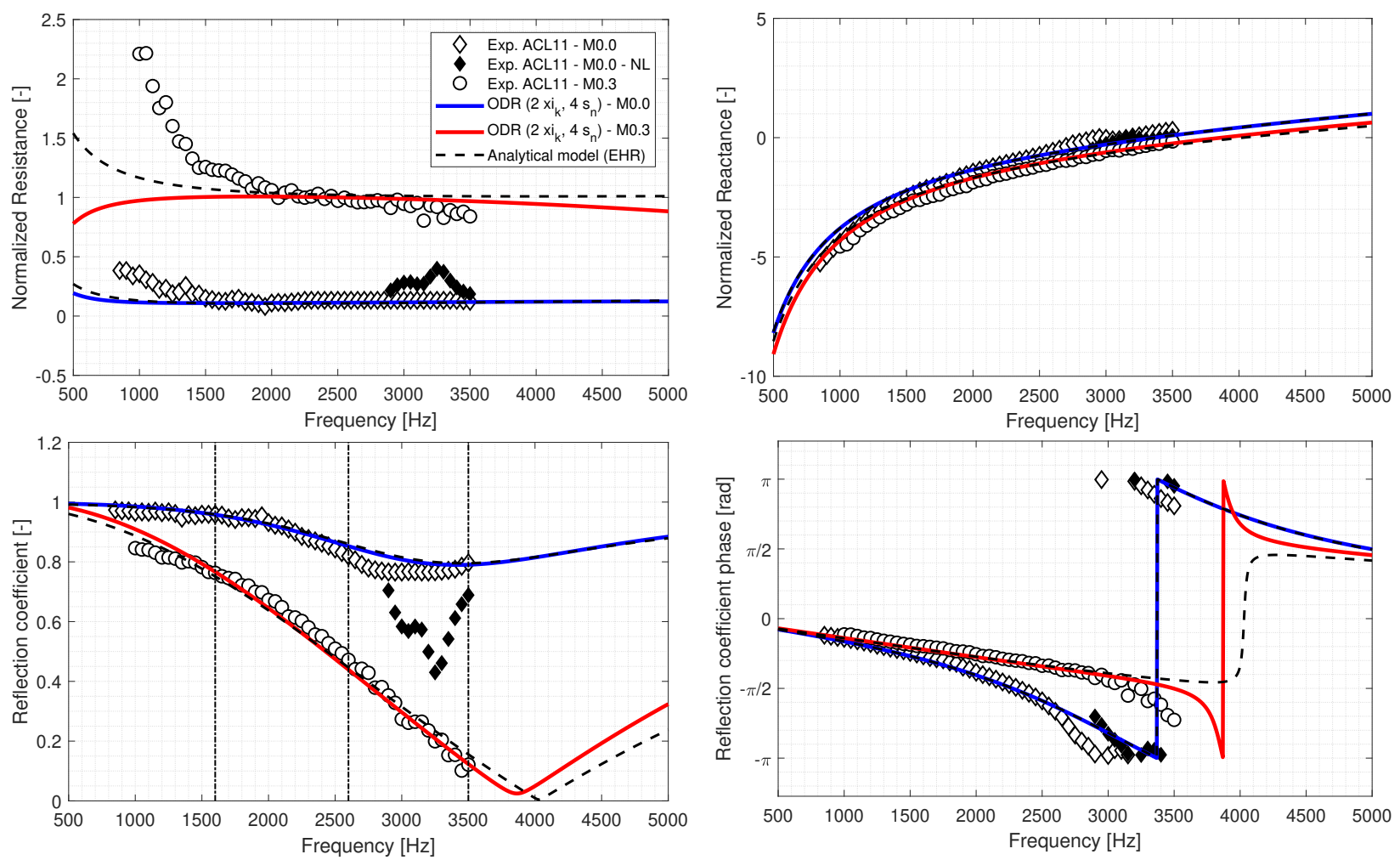

Fig. 6 Oscillo-Diffusive Representation of ACL Liner, 120dB, Mach 0.0 and Mach 0.3 
In AIB liner, the high resistance level derives from the wiremesh sheet, which is introducing a global higher resistive effect to overcome grazing flow sensibility. Indeed, if the Mach 0.3 flow is considered, the reflection coefficient module is practically unchanged. In ACL liner, the Mach 0 case shows a very low resistance and a nonlinear effect (black diamond symbols in Figure 6) at $3 \mathrm{kHz}$ due to resonance effect, as seen from null reactance at the same frequency. This physics can not be captured by the current linear impedance formulation. For simplicity, such nonlinear data have been approximated averaging the resistance level to nearby values (white diamond symbols). This approximation, applied on the range $2800-3500 \mathrm{~Hz}$, has shown negligible effects on the final results. In addition, the quiescent case shows a reflection coefficient close to 1 , which makes the liner's physics hard to be exactly reproduced, being a slight derivation from a hard wall. The grazing flow case presents a stronger reflection coefficient (lower in module), masking the nonlinear resonance effect identified in the Mach 0 case. Even if some discrepancies in the resistance level are present, the reflection coefficient module and phase are correctly discretized in the frequency range of interest, hence assuring an appropriate modelling in the CFD calculation. Differences in impedance laws due to SPL variations (up to $140 \mathrm{~dB}$ ) have shown to be negligible, which means that these liners are linear with respect to the SPL. Thus, only the impedance law at $120 \mathrm{~dB}$ is shown. To ensure numerical stability in the event of spurious oscillations appearing during the calculation, the impedance discretizations have been computed so to satisfy passivity, reality and causality conditions [30] up to $10 \mathrm{kHz}$. For both liners, 6 poles ( 2 real and 4 complex conjugated) are sufficient for a correct discretization, independently on the SPL and Mach number.

\section{CFD simulations setup}

Geometrical features retained for the numerical study are as following: a 3D cylinder of diameter $350 \mathrm{~mm}$, an acoustic treatment in the center of $175 \mathrm{~mm}$ and $350 \mathrm{~mm}$ for AIB and ACL liners respectively, and equally long hard walls of $325 \mathrm{~mm}$ upstream and downstream the liner. This brings to a ratio liner length over cylinder diameter of 0.5 and 1.0 and an overall duct length of $725 \mathrm{~mm}$ and $1000 \mathrm{~mm}$, for AIB and ACL configurations respectively. A structured multi-block grid is created with ANSA pre-processor ensuring minimal accuracy resolution needs: at least 30 points per wavelength in axial direction and 30 points in azimuthal and radial direction per highest relative mode 7 Three different grids have been created to verify the same level of accuracy at the three different frequencies. Two modal detection rings are replicated with two numerical surface outputs at the middle of each hard wall section, represented in Figure 4 by green lines, where instantaneous primitive variables are extracted at each iteration. Thanks to the acoustics toolbox Actran iTM, these values are converted into incident and reflected modes on each surface. It must not mislead the meaning of "incident" here. Taking as example the representation in Figure 7 incident and reflected modes on a surface correspond respectively to $\mathcal{A}_{L}^{+}$and $\mathcal{A}_{L}^{-}$for (left) Side $\mathrm{L}, \mathcal{A}_{R}^{+}$and $\mathcal{A}_{R}^{-}$for (right) Side R. When talking of incident and transmitted modes, it corresponds to $\mathcal{A}_{L}^{+}$and $\mathcal{A}_{R}^{+}$, if the acoustic waves propagate rightwards. For completion, in the same figure, $R_{L, m n}$ and $R_{R, m n}$ are the modal reflection coefficients (not to confuse with the impedance reflection coefficient $\beta$ ), expressing the relation between modes of a same section propagating on two opposite directions ( $m n$ and $\left.m^{\prime} n^{\prime}\right)$, and $T_{L, m n}, T_{R, m n}$ are the transmission coefficients, expressing the relation between two modes of two different sections ( $\mathrm{L}$ and $\mathrm{R}$ ) propagating in the same direction.

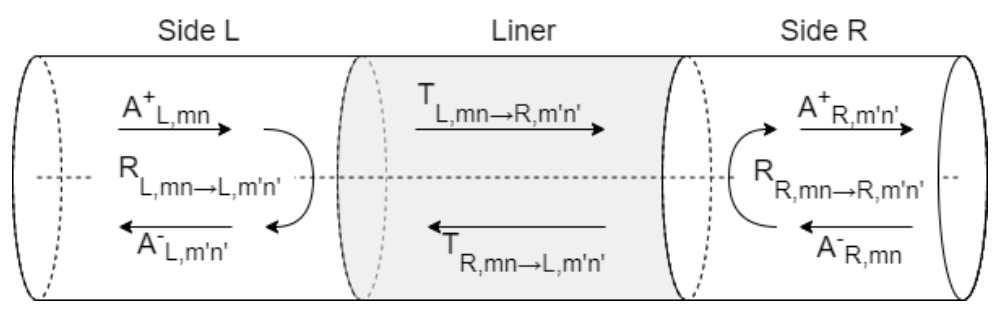

Fig. 7 Modes decomposition in a cylindrical duct

Experimental results are given in terms of Transmission Loss (TL), the direct difference in decibels between incident and transmitted modes in the treated case. During the experiment, hard-walled configurations were run for each mode to verify that in this case the TL was null, hence no acoustic losses were found. In the present numerical study, only few configurations were tested in a hard-wall layout for the same verification purpose, which proved confirmed. As already introduced, loudspeakers could control the azimuthal order injected in the experiment (through a phase controlling

\footnotetext{
${ }^{\dagger}$ Example, for a mode m3n2, minimum 90 and 60 points in azimuthal and radial directions, respectively
} 
process), but not the radial order. For this reason, each experimental run was done at a given azimuthal order only. Modal detection can provide the distribution on all propagative modes (azimuthal and radial) of a given injected signal. However, phase information of the injected signal was not available. To overcome these limitations, a recombination on the actual experimental injected modes $(m, n)$ is carried out through a weighting-like post-processing. Practically, CFD simulations are run by imposing a constant pressure amplitude $\mathcal{A}_{m n}$ of $120 \mathrm{~dB}$ for a specific azimuthal and radial mode $(m, n)$. This means having a different injected intensity level for each mode, since the latter does not scale with $\mathcal{A}_{m n}$ but is also function of the modal shape:

$$
<I_{m n}>=\mathcal{A}_{m n}^{2} \frac{\pi}{S \rho_{0} c_{0}} \frac{N_{m n} \Psi_{m n}^{ \pm}}{\left|k-M k_{z, m n}^{ \pm}\right|^{2}}
$$

where $S$ is the reference surface (i.e. the circular section), $N_{m n}$ and $\Psi_{m n}$ are parameters linked to the modal structure (given in Appendix) and " $<>$ " is the spatially averaged value. Under the assumption of linear acoustics, the intensity levels can then be scaled to those detected in the experiment. In order to satisfy this recombination, it is impossible to run CFD simulations including all radial orders of a single azimuthal order as in the experiment, and each mode has to be injected singularly. In the following, intensity variables $I$ are considered spatially averaged intensities $\langle I\rangle$ as given by [29) and taken in their dimensional form $\left[W / m^{2}\right]$ for avoiding logarithmic expressions. A scaling factor $I_{(m, n)}^{S F}$ for each injected mode $(m, n)$ is defined as the ratio between the intensity of incident modes in the experiment $I_{(m, n)}^{\text {exp,inc }}$ and the one computed in the CFD $I_{(m, n)}^{\mathrm{CFD} \text {,inc. }}$

$$
I_{m n}^{\mathrm{SF}}=\frac{I_{m n}^{\mathrm{exp}, \mathrm{inc}}}{I_{m n}^{\mathrm{CFD}, \mathrm{inc}}}
$$

Thereby, the transmitted intensity level after the treatment is scaled with this factor to be comparable to the experimental acoustic level. For each simulation run at mode $(m, n)$, all the contributions of propagative modes $\left(m, n^{\prime}\right)$ are taken into account. As saying, when injecting the mode $(0,2)$, the transmitted propagative modes $(0,1),(0,2),\left(0, n^{\prime}\right)$ will be considered in the scaled transmitted intensity $I_{(m, n)}^{\mathrm{sc}, \mathrm{trn}}$ :

$$
I_{m n}^{\mathrm{sc}, \mathrm{trn}}=I_{m n}^{\mathrm{SF}} \sum_{n^{\prime}} I_{\left.m n n^{\prime}\right)}^{\mathrm{CFD}, \mathrm{trn}}
$$

Finally, the Transmission Loss can be computed as the difference (in logarithm) of all propagative modes of a specific azimuthal order:

$$
T L_{(m)}=10 \log _{10}\left(\frac{\sum_{n} I_{m n}^{\mathrm{exp}, \mathrm{inc}}}{\sum_{n} I_{m n}^{\mathrm{sc}, \mathrm{trn}}}\right)
$$

Each simulation is run with a temporal resolution of 100 timesteps per acoustic period for a total of 30 periods of time. The last period is then extracted and post-processed for modal decomposition. A sensitivity study was conducted on the number of periods needed to reach a converged TL, and one period showed to be sufficient for most cases.

\section{CFD results on 3D cylinder}

First, a qualitative analysis in Figure 8 shows a converged mode $\mathrm{m} 5 \mathrm{n} 2$ propagation and attenuation in the cylindrical duct. The rotating acoustic pattern injected on the left side propagates spiraling rightwards, it is attenuated in amplitude through the central lined section and ejected from the right side. Few interesting acoustic structures appeared, such as periodic "circles" that can be seen on the lateral surface. They are thought caused by reflections from the impedance wall discontinuity. This generates oscillations in the sound level (SPL) preceding the liner, as also proven by 2D results in [26].

Next, the Transmission Losses (TL) are evaluated. The results herein obtained with elsA CFD software are compared with the experiment [34] and the recent achievements of Nair in [25] with the time-based CAA code Actran-DGM solving Linearized Euler Equations. Both numerical results are obtained adopting the same acoustic treatments showed in Figures 5 and 6 . This fruitful comparison allowed a higher fidelity validation of both numerical implementations, rather than solely basing it on the experimental measurements. Although no comprehensive uncertainty study has been performed on this very complex bench, the repeatability has been assessed below $1 \mathrm{~dB}$, apart for unexplained instabilities on some modes and at high Mach. The overall accuracy can be thus estimated around $1 \mathrm{~dB}$ on the selected 


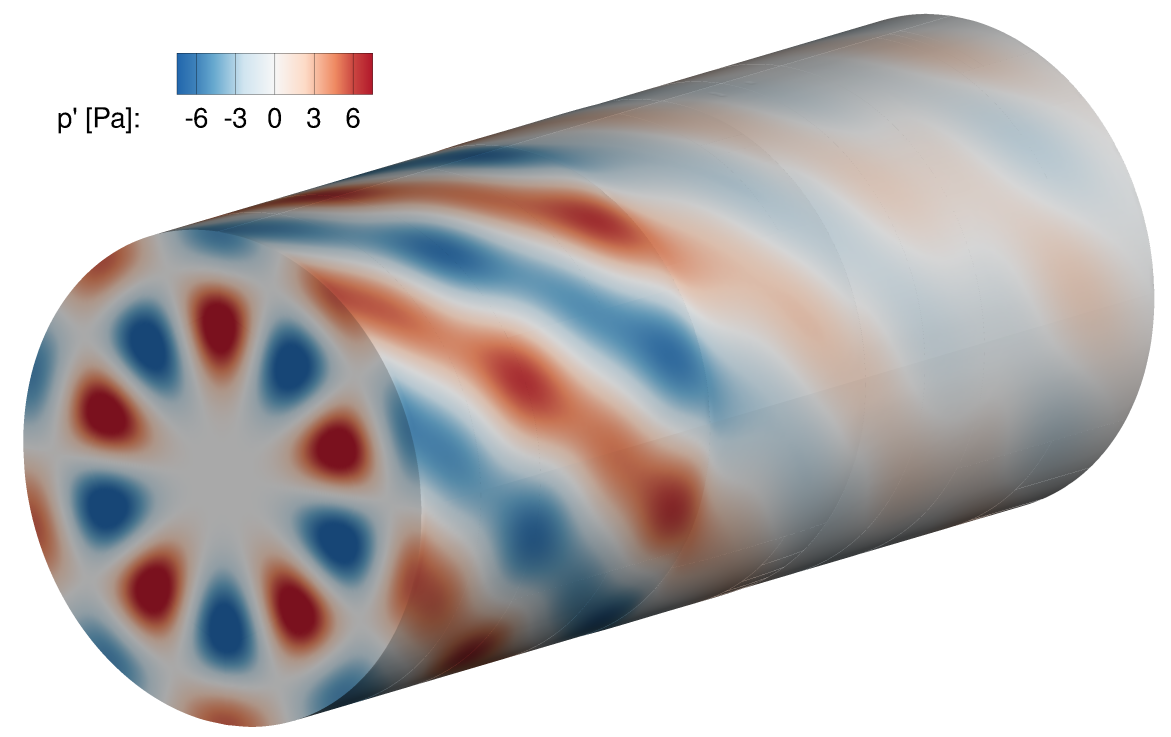

Fig. 8 High-order mode $(\mathbf{m} 5 \mathrm{n} 2)$ propagation and attenuation in CANNELLE CFD test case.

measurement points used in this paper. In all the following analyses, the CAA-LEE numerical results will be taken as the reference for two reasons. First, because this code showed to be accurate on several academic and industrial validations. Second and as a reminder of the present development's objective, to show the capabilities of a CFD software to reproduce what is usually achieved with a CFD/CAA coupled technique. Figures 9 shows the Transmission Losses for the AIB liner at Mach 0, for frequencies of 1600, 2600 and $3500 \mathrm{~Hz}$. Good coherence is found between the two codes (maximum deviation of $2 \mathrm{~dB}$ ) for most of the modes at all frequencies, with only few cases at high azimuthal order where a slight higher attenuation from the CFD is found. These cases are also presenting the strongest differences with the experiment (however lower than $4 \mathrm{~dB}$ ), but given the difficulty in reproducing such complex acoustics environment, both experimental and numerical results can be considered a close representation of the reality. For time constraints, the inlet configuration case at Mach 0.3 is not tackled at this stage and will be subject of future developments.

Similar considerations are drawn for the ACL case at Mach 0, shown in Figure 10. Stronger differences are found for high order modes, where elsA showed stronger attenuation levels. However, an overall satisfying precision was found in lower order modes between the two codes. Wider differences are found against the experiment, but it must be reminded that this SDOF liner is conceived to operate in a grazing flow condition, making experimental and numerical results without flow not completely reliable.

A preliminary analysis with flow at Mach 0.3 is shown in Figure 11. This first analysis considers an Euler flow (no viscosity effects) and a prescribed constant flow profile, hence no boundary layer interaction with the lined wall. This is a strong simplification that brings non negligible differences at high order modes when compared to CAA, where LEE are solved with a prescribed boundary layer as in the experimental measurements. In addition, the numerical grid have been maintained the same, hence not considering the flow effect on wavelength modification. The results are however promising and some conclusions can be drawn. The addition of an air flow modifies cut-off frequencies, hence having more propagative modes. An added flow also brings a stronger sound attenuation (up to $50 \mathrm{~dB}$ ), as expected by looking at the lower reflection coefficient module in Figure 6. Given the closer accuracy of CAA to the experiment, the inclusion of a shear boundary layer is thought to have a strong impact on the results, and that once taken into account it will bring CFD results in closer alignment as in the quiescent cases previously detailed.

\section{Conclusion}

The Oscillo-Diffusive Representation already showed to be a powerful tool in acoustic absorption problems. Its implementation in an industrial CFD code through a Time-Domain Impedance Boundary Condition with a characteristics formalism has been detailed. The present work brings an extended validation on high-order acoustic modes attenuation in a 3D case. Regardless the complex numerical layout and the rich acoustics content, the boundary condition showed to be successfully implemented in the CFD code, providing encouraging results in close alignment with an aeroacoustics 




Fig. 9 Transmission Losses of AIB liner - Mach 0

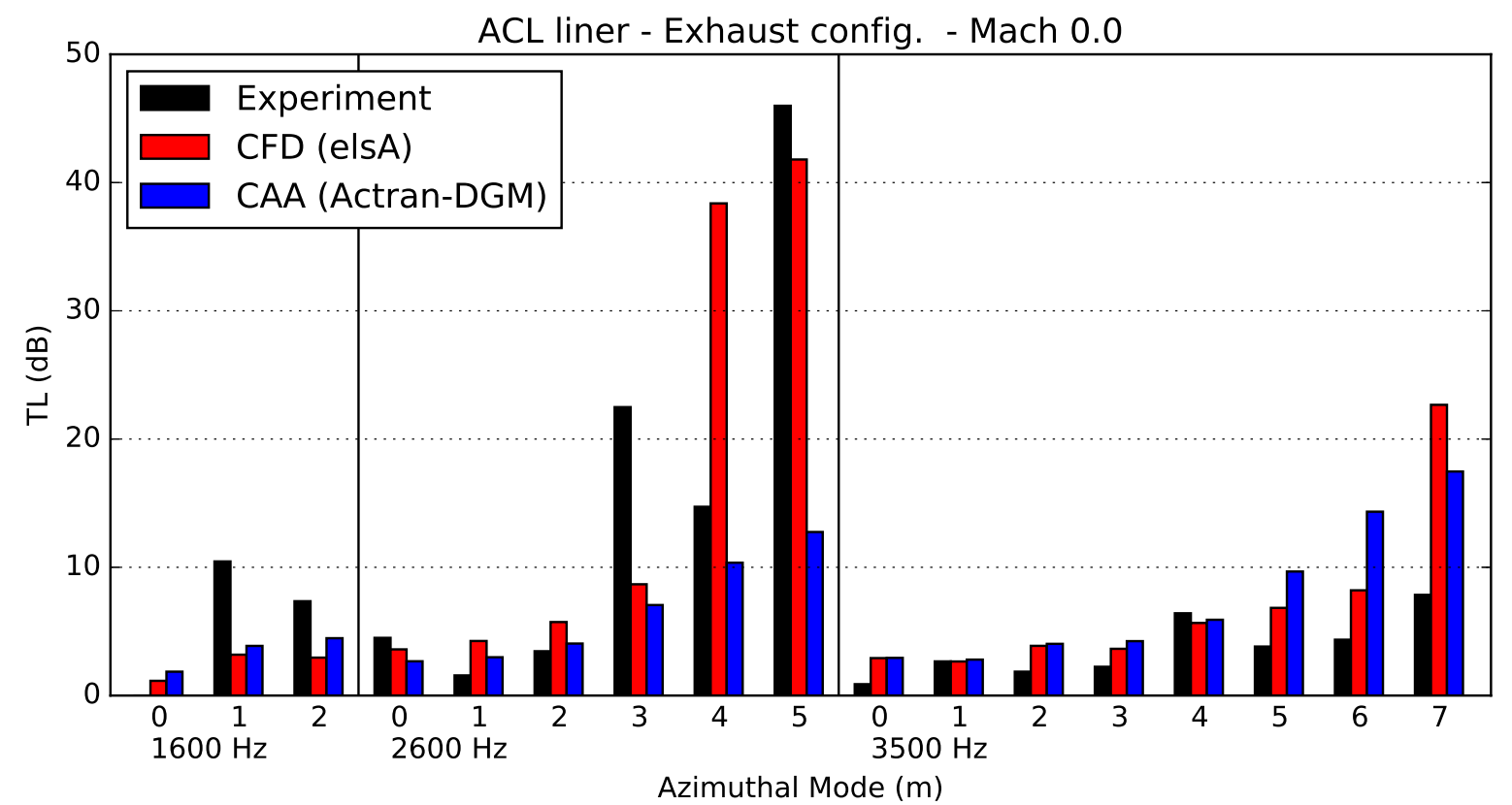

Fig. 10 Transmission Losses of ACL liner - Mach 0

code and experiment. Further studies are expected to be carried out to improve these results at high azimuthal orders. Notably the inclusion of Navier-Stokes equations with boundary layer in exhaust and inlet configurations, a detailed grid convergence analysis in presence of flow and a time-space convergence study for high order modes. Nevertheless, time-domain liner attenuation in a CFD code as alternative to a coupled CFD/CAA process has been proven applicable in complex acoustics environments. A future extension to a fan inlet configuration is foreseen, in presence of strong nonlinear acoustic waves which can be resolved by a CFD code and that are expected to be correctly attenuated by the broadband ODR impedance model. 


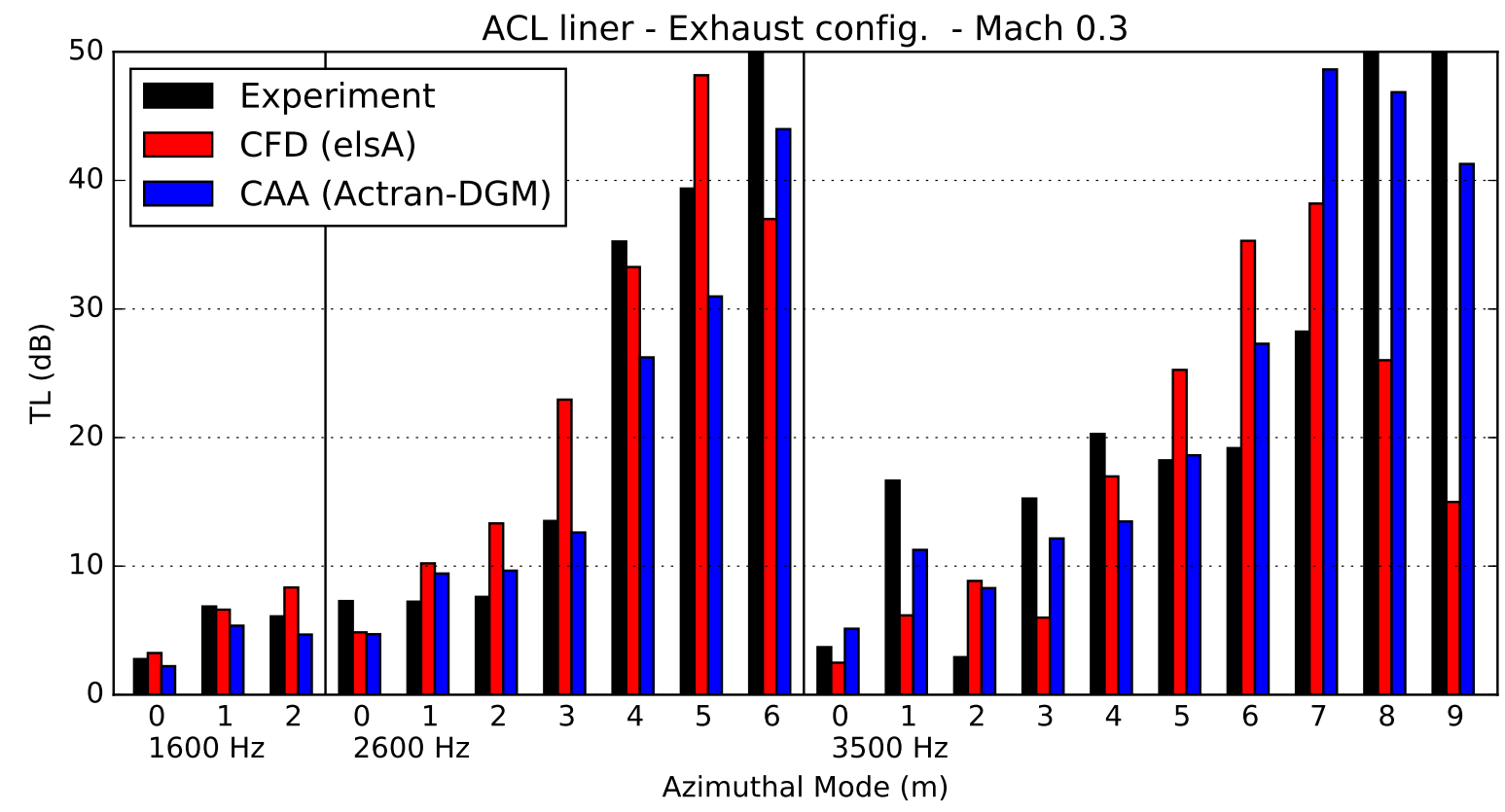

Fig. 11 Transmission Losses of ACL liner - Mach 0.3

\section{Acknowledgments}

The first author thanks Airbus Operations S.A.S. and ANRT for financing the work under the Ph.D. CIFRE contract $\mathrm{n}^{\circ}$ 2018/0772, Onera for the fundamental technical support, Johan Thisse and Guilherme Coelho Cunha (Airbus) for their valuable guidance. The authors are grateful to Maud Lavieille (Airbus) for providing extensive material on the CANNELLE bench, and to Mehdi Naïr (Free Field Technologies) for the fruitful exchanges and for sharing data from the Actran simulations. The authors are also grateful to Safran Nacelles for providing information on their liner ACL considered for validating the present work. The solver used herein is elsA (ensemble logiciel de simulation aérodynamique, software package for aerodynamic simulation), jointly owned by ONERA, Safran and Airbus.

\section{Appendix}

The pressure fluctuation for a unitary modal amplitude and phase $(\phi)$ is given function of the cylindrical coordinates $(r, \theta, z)$ and the modal structure $\left(m, n, k_{r, m n}\right)$ as:

$$
p_{m n}^{ \pm}(z, r, \phi, t)=\left(B_{m n} J_{m n}\left(k_{r, m n} r\right)+C_{m n} Y_{m n}\left(k_{r, m n} r\right)\right) e^{i\left(\omega t+m \phi-k_{z, m n}^{ \pm} z\right.}
$$

with \pm defining if the propagation is on the positive or negative axis, $J_{m}$ and $Y_{m}$ the Bessel functions of first and second species, $J_{m}^{\prime}$ and $Y_{m}^{\prime}$ their derivatives,

$$
B_{m n}=\cos \left(\Theta_{m n}\right), \quad C_{m n}=\sin \left(\Theta_{m n}\right), \quad \Theta_{m n}=\arctan \left(-\frac{J_{m}^{\prime}\left(k_{r, m n} R_{e x t}\right)}{Y_{m}^{\prime}\left(k_{r, m n} R_{\text {ext }}\right)}\right)
$$

with $k_{r, m n}$ the radial wavenumber (real positive), solution of (in the case of hard-walled duct):

$$
\left\{J_{m}^{\prime}\left(k_{r, m n} R_{\text {int }}\right)\right\} \times\left\{Y_{m}^{\prime}\left(k_{r, m n} R_{\text {ext }}\right)\right\}-\left\{Y_{m}^{\prime}\left(k_{r, m n} R_{\text {int }}\right)\right\} \times\left(J_{m}^{\prime}\left(k_{r, m n} R_{\text {ext }}\right)\right)=0
$$

with $k_{z, m n}$ the axial wavenumber, which is real for propagative modes and imaginary for cut-off (evanescent) modes:

$$
k_{z, m n}^{ \pm}=\frac{k}{1-M^{2}}\left(-M \pm \sqrt{1-\left(1-M^{2}\right)\left(\frac{k_{r, m n}}{k}\right)^{2}}\right)
$$


The coefficient $N_{m n}$ used to calculate the modal intensity in 29 is given by:

$$
\begin{aligned}
N_{m n} & =\int_{R_{i n t}}^{R_{e x t}} r\left[B_{m n} J_{m n}\left(k_{r, m n} r\right)+C_{m n} Y_{m n}\left(k_{r, m n} r\right)\right]^{2} d r= \\
& =\frac{1}{2}\left[\left(r^{2}-\frac{m^{2}}{k_{r, m n}^{2}}\right)\left[B_{m n} J_{m n}\left(k_{r, m n} r\right)+C_{m n} Y_{m n}\left(k_{r, m n} r\right)\right]^{2}\right]_{r=R_{i n t}}^{r=R_{e x t}}
\end{aligned}
$$

and the modal shape $\Psi_{m n}$ is the real part of:

$$
\Psi_{m n}=\mathcal{R e}\left(M k^{2}+k\left(k_{z, m n}^{ \pm *}-M^{2} k_{z, m n}^{ \pm}\right)\right)
$$

\section{References}

[1] Casalino, D., Hazir, A., and Mann, A., "Turbofan Broadband Noise Prediction Using the Lattice Boltzmann Method," AIAA Journal, Vol. 56, No. 2, 2018, pp. 609-628. https://doi.org/10.2514/1.J055674

[2] Tam, C. K. W., and Auriault, L., "Time-domain impedance boundary conditions for computational aeroacoustics," AIAA Journal, Vol. 34, No. 5, 1996, pp. 917-923. https://doi.org/10.2514/3.13168.

[3] Rienstra, S., "Impedance Models in Time Domain, Including the Extended Helmholtz Resonator Model," Collection of Tech. Papers - 12th AIAA/CEAS Aeroacoustics Conference, Vol. 6, 2006. https://doi.org/10.2514/6.2006-2686

[4] Fung, K., and Ju, H., "Broadband Time-Domain Impedance Models,” AIAA Journal, Vol. 39, No. 8, 2001 , pp. 1449-1454. https://doi.org/10.2514/2.1495.

[5] Reymen, Y., Baelmans, M., and Desmet, W., "Efficient Implementation of Tam and Auriault's Time-Domain Impedance Boundary Condition,” AIAA Journal, Vol. 46, No. 9, 2008, pp. 2368-2376. https://doi.org/10.2514/1.35876.

[6] Li, X. Y., Li, X. D., and Tam, C. K. W., "Improved Multipole Broadband Time-Domain Impedance Boundary Condition,” AIAA Journal, Vol. 50, No. 4, 2012, pp. 980-984. https://doi.org/10.2514/1.J051361

[7] Troian, R., Dragna, D., Bailly, C., and Galland, M.-A., "Broadband liner impedance eduction for multimodal acoustic propagation in the presence of a mean flow," Journal of Sound and Vibration, Vol. 392, 2017, pp. 200-216. https: //doi.org/10.1016/j.jsv.2016.10.014

[8] Monteghetti, F., Matignon, D., and Piot, E., "Energy analysis and discretization of nonlinear impedance boundary conditions for the time-domain linearized Euler equations," Journal of Computational Physics, Vol. 375, 2018, pp. 393 - 426. https://doi.org/10.1016/j.jcp.2018.08.037

[9] Monteghetti, F., Matignon, D., Piot, E., and Pascal, L., "Design of broadband time-domain impedance boundary conditions using the oscillatory-diffusive representation of acoustical models," The Journal of the Acoustical Society of America, Vol. 140, 2016, pp. 1663-1674. https://doi.org/10.1121/1.4962277

[10] Gabard, G., and Brambley, E., "A full discrete dispersion analysis of time-domain simulations of acoustic liners with flow," Journal of Computational Physics, Vol. 273, 2014, pp. 310 - 326. https://doi.org/10.1016/j.jcp.2014.05.004

[11] Dragna, D., Pineau, P., and Blanc-Benon, P., "A generalized recursive convolution method for time-domain propagation in porous media," The Journal of the Acoustical Society of America, Vol. 138, No. 2, 2015, pp. 1030-1042. https: //doi.org/10.1121/1.4927553

[12] Liu, X., Huang, X., and Zhang, X., "Stability analysis and design of time-domain acoustic impedance boundary conditions for lined duct with mean flow," The Journal of the Acoustical Society of America, Vol. 136, No. 5, 2014, pp. $2441-2452$. https://doi.org/10.1121/1.4896746.

[13] Zhong, S., Zhang, X., and Huang, X., "A Controllable Canonical Form Implementation of Time Domain Impedance Boundary Conditions for Broadband Aeroacoustic Computation,” Journal of Computational Physics, Vol. 313, No. C, 2016, pp. 713-725. https://doi.org/10.1016/j.jcp.2016.03.002

[14] Van den Nieuwenhof, B., and Coyette, J.-P., "Treatment of frequency-dependent admittance boundary conditions in transient acoustic finite/infinite-element models," The Journal of the Acoustical Society of America, Vol. 110, 2001, pp. $1743-51$. https://doi.org/10.1121/1.1404436. 
[15] Douasbin, Q., Scalo, C., Selle, L., and Poinsot, T., "Delayed-time domain impedance boundary conditions (D-TDIBC)," Journal of Computational Physics, Vol. 371, 2018. https://doi.org/10.1016/j.jcp.2018.05.003.

[16] Delorme, P., Mazet, P., Peyret, C., and Ventribout, Y., "Computational aeroacoustics applications based on a discontinuous Galerkin method," Comptes Rendus Mécanique, Vol. 333, No. 9, 2005, pp. 676-682. https://doi.org/10.1016/j.crme.2005.07.007

[17] Delattre, G., Sagaut, P., Manoha, E., and Redonnet, S., “Time-Domain Simulation of Sound Absorption on Curved Wall," 13th AIAA/CEAS Aeroacoustics Conference (28th AIAA Aeroacoustics Conference), 2007. https://doi.org/10.2514/6.2007-3493

[18] Escouflaire, M., Redonnet, S., and Aurégan, Y., "Further Insights on Time-Domain Impedance Boundary Condition," 19th AIAA/CEAS Aeroacoustics Conference, 2013. https://doi.org/10.2514/6.2013-2219.

[19] Escouflaire, M., "Theoretical and Numerical Investigation of Time-Domain Impedance Models for Computational Aeroacoustics," Ph.D. dissertation, Académie de Nantes, 2014.

[20] Scalo, C., Bodart, J., and Lele, S. K., "Compressible turbulent channel flow with impedance boundary conditions," Physics of Fluids, Vol. 27, No. 3, 2015, p. 035107. https://doi.org/10.1063/1.4914099

[21] Burak, M. O., Billson, M., Eriksson, L.-E., and Baralon, S., "Validation of a Time- and Frequency-Domain Grazing Flow Acoustic Liner Model," AIAA Journal, Vol. 47, No. 8, 2009, pp. 1841-1848. https://doi.org/10.2514/1.40870.

[22] Fiévet, R., Deniau, H., and Piot, E., "Strong compact formalism for characteristic boundary conditions with discontinuous spectral methods," Journal of Computational Physics, Vol. 408, 2020, pp. 1-26. https://doi.org/10.1016/j.jcp.2020.109276

[23] Fiévet, R., Deniau, H., Brazier, J.-P., and Piot, E., Numerical Study of Hypersonic Boundary-Layer Transition Delay through Second-Mode Absorption, 2020. https://doi.org/10.2514/6.2020-2061

[24] Shur, M., Strelets, M., Travin, A., Suzuki, T., and Spalart, P. R., Unsteady Simulation of Sound Propagation in Turbulent Flow Inside a Lined Duct Using a Broadband Time-Domain Impedance Model, 2020. https://doi.org/10.2514/6.2020-2535.

[25] Naïr, M., de Brye, B., Legendre, C., Casadei, L., and Cunha, G., "Industrial-scale time domain modelling of acoustic surface treatments for aero-engines using discontinuous Galerkin method," 2021 AIAA AVIATION Forum and Exposition (submitted), 2021.

[26] Casadei, L., Deniau, H., Piot, E., and Nodé-Langlois, T., “Time-Domain Impedance Boundary Condition Implementation in a CFD solver and validation against experimental data of acoustical liners," eForum Acusticum, 2020, pp. 359-366. https://doi.org/10.48465/fa.2020.0088

[27] Zaabar, K., and Wilson, A. G., A Non-reflective Boundary Condition for Prediction of Acoustic Tones in Turbomachinery using Computational Fluid Dynamics, 2020. https://doi.org/10.2514/6.2019-2747.

[28] Giles, M. B., "Nonreflecting boundary conditions for Euler equation calculations," AIAA Journal, Vol. 28, No. 12, 1990, pp. 2050-2058. https://doi.org/10.2514/3.10521.

[29] Poinsot, T., and Lele, S., "Boundary conditions for direct simulations of compressible viscous flows," Journal of Computational Physics, Vol. 101, No. 1, 1992, pp. 104 - 129. https://doi.org/10.1016/0021-9991(92)90046-2

[30] Rienstra, S., and Hirschberg, A., An introduction to acoustics, Eindhoven University of Technology, 2021.

[31] Bruneau, M., Fundamental of Acoustics, London: ISTE Ltd, 2006.

[32] Crandall, I. B., Theory of Vibrating Systems and Sound, D. Van Nostrand Company, 1926.

[33] Hixon, R., Nallasamy, R., Sawyer, S., and Dyson, R., "Mean Flow Boundary Conditions for Computational Aeroacoustics," 2003. https://doi.org/10.2514/6.2003-3299

[34] Lavieille, M., and Le-Saint, S., Impedance eduction of liners in no-flow condition and based on multimodal excitation, 2017. https://doi.org/10.2514/6.2017-3180 